\title{
Tunneling and the Onset of Chaos in a Driven Bistable System
}

\author{
R. Utermann, T. Dittrich, and P. Hänggi \\ Institut für Physik, Universität Augsburg, Memminger Straße 6, D-86135 Augsburg, Germany
}

(October 30, 2018)

\begin{abstract}
We study the interplay between coherent transport by tunneling and diffusive transport through classically chaotic phase-space regions, as it is reflected in the Floquet spectrum of the periodically driven quartic double well. The tunnel splittings in the semiclassical regime are determined with high numerical accuracy, and the association of the corresponding doublet states to either chaotic or regular regions of the classical phase space is quantified in terms of the overlap of the Husimi distribution with the chaotic layer along the separatrix. We find a strong correlation between both quantities. They show an increase by orders of magnitude as chaotic diffusion between the wells starts to dominate the classical dynamics. We discuss semiclassical explanations for this correlation.
\end{abstract}

05.45.+b, 03.65.Sq, 73.40.Gk

Typeset using REVTEX 


\section{INTRODUCTION}

In mesoscopic systems with a bistable potential, two fundamentally different modes of transport can occur simultaneously: Coherent quantal transport by tunneling, and deterministic diffusion through chaotic regions interweaving the classical phase space. There is a strong mutual influence of these two processes. While the classical phase-space structure is clearly reflected in the quantal spectrum [1] [-3], localization and delocalization due to quantal interference, in turn, effectively alter the boundary conditions for the quantal counterpart of classical phase-space diffusion [4]10].

The idea that chaotic layers separating symmetry-related regular regions can give rise to quantal tunneling, just as potential barriers do, has been introduced by Davis and Heller [7], who dubbed this notion "dynamical tunneling". The fingerprint of dynamical tunneling in the spectrum has been studied by Bohigas et al. [8,9]. They utilized the occurrence of tunnel doublets with exponentially small splittings as a filter to separate "regular" quantal eigenstates (i.e., those localized in classically regular phase-space regions) from "chaotic" ones (in an analogous sense). While their work was based on a time-independent, twodimensional nonlinear oscillator, the first inquiry into driven tunneling in a one-dimensional, bistable system was undertaken by Lin and Ballentine [11] and Grossmann et al. [12 14], and subsequently by Plata and Gomez Llorente [15].

Up to now, the only attempt towards an analytical understanding of tunneling between quantum states localized on symmetry-related tori (or chaotic regions) in multi-dimensional systems has been made by Wilkinson and Hannay 16,17. Using semiclassical methods, they express the tunnel splitting as a function of the classical action along paths connecting the classical manifolds that support the doublet states. However, their theory does not provide a unified description spanning the transition from regular to chaotic states. It is this transition which particularly interests us here.

Our present work (a preliminary account of which has been published in ref. [18]) is similar in scope to that of Bohigas et al. mentioned above, but devoted to a different sys- 
tem class. Applying torus or EBK (Einstein-Brillouin-Keller) quantization for periodically driven systems [19] as well as the random-matrix theory for mixed (regular and chaotic) systems [8]20] to the Floquet spectrum of the periodically driven double well, we expect the following scenario: The classical phase space of this system, for not too strong driving, is characterized by a pair of symmetry-related regular regions near the minima of the unperturbed potential, embedded in a chaotic layer along the unperturbed separatrix. Quantal eigenstates localized in the regular regions form tunnel doublets with exponentially small splittings. These doublets will break up, and their splittings will reach values of the order of the mean level spacing, as soon as the associated pair of quantizing tori dissolves in the chaotic layer. Therefore, the tunnel splitting should depend strongly on the nature - regular or chaotic - of the classical phase-space region to which the corresponding pair of eigenstates predominantly belongs. In order to allow for a numerical test of this conclusion, we shall quantify this association in terms of the overlap of a suitable phase-space representation of the eigenstates with the chaotic layer.

We emphasize that our hypothesis relies crucially on the specific phase-space topology of driven bistable systems. That is, the decisive property that prevents states within the chaotic layer from forming tunnel doublets is not a positive local Lyapunov exponent of the classical dynamics, but rather the possibility of phase-space transport across the symmetry plane. For example, in the converse case - symmetry-related chaotic islands embedded in regular regions - one expects tunnel-splitted level pairs which, in turn, repel each other in the same way as the single levels of the individual chaotic regions would do. Furthermore, it should be kept in mind that the disintegration of a classical torus is not an abrupt all-ornothing event. Rather, this invariant manifold passes through a fractal, intermediate stage ("cantorus" [21]) before it disappears, and even after that, a "shadow" remains in the form of a distinct, repelling structure within the chaotic sea ("vague torus" [22]). Due to the finite phase-space resolution of the quantal dynamics, this transition is smeared out even further in the parameter space of the corresponding quantum system.

We give a brief review of our working model, its symmetries and classical dynamics, in 
Section [1]. In Section [II, we introduce the methods and key quantities used in our numerical study and interpret our results on basis of the simple picture just sketched. Section $\mathbb{\square}$ contains the summary and puts our findings in a broader perspective.

\section{THE MODEL: SYMMETRIES AND CLASSICAL DYNAMICS}

The harmonically driven quartic double well is described by the Hamiltonian

$$
\begin{aligned}
H(x, p ; t) & =H_{0}(x, p)+H_{1}(x ; t), \\
H_{0}(x, p) & =\frac{p^{2}}{2}-\frac{1}{4} x^{2}+\frac{1}{64 D} x^{4}, \\
H_{1}(x ; t) & =x S \cos (\omega t) .
\end{aligned}
$$

With the dimensionless variables used, the only parameter controlling the unperturbed Hamiltonian $H_{0}(x, p)$ is the barrier height $D$. It can also be interpreted as the (approximate) number of doublets with energies below the top of the barrier. Accordingly, the classical limit amounts to letting $D \rightarrow \infty$. The driving is characterized by its amplitude $S$ and frequency $\omega$. In all our numerical studies, we kept barrier height and driving frequency fixed at the values $D=8$ and $\omega=0.95$, respectively.

Besides invariance under time-reversal, $x \rightarrow x, p \rightarrow-p, t \rightarrow-t$, the unperturbed system possesses the spatial reflection symmetry $x \rightarrow-x, p \rightarrow p, t \rightarrow t$. For a general periodic driving, this twofold symmetry is destroyed and replaced by the discrete timetranslation invariance under $t \rightarrow t+2 \pi / \omega$. On the quantum-mechanical level, this enables the application of the Floquet formalism 223 26]. For the specific time dependence of a harmonic driving, the symmetry $f(t+\pi / \omega)=-f(t)$ restores a similar situation as in the unperturbed case: The system is now invariant against the operation 12, 14, 27.

$$
\mathrm{P}: p \rightarrow-p, \quad x \rightarrow-x, \quad t \rightarrow t+\frac{\pi}{\omega}
$$

which may be regarded as a generalized parity in the extended phase space spanned by $x, p$, and phase, i.e., time $t \bmod (2 \pi / \omega)$. As in the unperturbed case, this enables to separate the 
eigenstates into an even and an odd subset. Moreover, as far as the dynamics allows, the generalized parity gives rise to tunnel doublets with the corresponding pair of eigenstates residing within the vortex tubes [19] formed by the motion within the potential wells of the unperturbed system. These tunnel doublets, in particular, are the subject of our study.

The unperturbed system, as given by $H_{0}(x, p)$, exhibits the generic phase space of a bistable system (Fig. 1a). Its structure is even independent, up to a rescaling of $x$ and $p$, of the parameter $D$, provided $D>0$. Apart from a constant of the order of unity, $D$ gives the phase-space area enclosed by the two loops of the separatrix. It represents the characteristic action of the unperturbed system to be compared, in particular, with $\hbar$.

For the parameter values of the driving that are relevant for the present study, $\omega=$ 0.95 and $0 \leq S \leq 0.2$, the phase space of the unperturbed system is modified mainly by two additional features [28]: The onset of chaos in the vicinity of the separatrix, and the growth of the first nonlinear resonance zone (Figs. 1b,c). The chaotic layer along the separatrix develops out of the homoclinic tangle, the intricate interweaving of the stable and the unstable manifolds originating at the hyperbolic fixed point near the top of the barrier [29]. It is present for all $S>0$, but covers a substantial part of phase space only for $S \gtrsim 0.1$.

Nonlinear resonance arises when (an integer multiple $k$ of) the period of the driving equals the period of the oscillation within each well at some energy. Each resonance generates a chain of $k$ alternating elliptic and hyperbolic fixed points, embedded in the regular regions within each well. For example, the vortex tube surrounding, in the extended phase space, the elliptic fixed point of the first resonance, winds around the vortex tube corresponding to nonresonant motion once per period of the driving. Within the parameter interval $10^{-3} \lesssim$ $S \lesssim 10^{-2}$, this first resonance zone grows from near invisibility to almost the full area of the regular region within each well. The higher resonances occupy only extremely narrow filaments in phase space which accumulate at the top of the barrier. Therefore, the border zone between the chaotic layer and the regular regions, formed by the higher resonances, is also very narrow, and in fact comes close to a sharply defined borderline [30]. As a further consequence, the chaotic layer "gains ground" in a quite smooth manner, as $S$ increases. 
The intricate, self-similar layering of the border zone implied by the KAM theorem [29] does not visibly affect this process.

For larger values of the driving amplitude, higher-order features, such as secondary islands, appear and render the phase-space structure increasingly complicated (Fig. 1d). As we shall see below, the growth of the first resonance leaves no significant trace in the tunnel splittings, while the consequences of the spreading of the chaotic layer are drastic.

\section{NUMERICAL METHODS AND RESULTS}

The basic ingredient required to study the quantum-mechanical aspects of a periodically driven system is the Floquet operator [23 26], i.e., the unitary propagator that generates the time evolution over one period of the driving force,

$$
U=\mathrm{T} \exp \left(-\frac{\mathrm{i}}{\hbar} \int_{0}^{2 \pi / \omega} \mathrm{d} t H(t)\right)
$$

where $\mathrm{T}$ denotes time ordering. Its eigenvectors and eigenphases, referred to as Floquet states and quasienergies, respectively, can be written in the form

$$
\left|\psi_{\alpha}(t)\right\rangle=\mathrm{e}^{-\mathrm{i} \epsilon_{\alpha} t}\left|\phi_{\alpha}(t)\right\rangle
$$

with

$$
\left|\phi_{\alpha}(t+2 \pi / \omega)\right\rangle=\left|\phi_{\alpha}(t)\right\rangle
$$

From a Fourier expansion of the $\left|\phi_{\alpha}(t)\right\rangle$,

$$
\begin{aligned}
\left|\phi_{\alpha}(t)\right\rangle & =\sum_{n}\left|c_{\alpha, n}\right\rangle \mathrm{e}^{-\mathrm{i} n \omega t}, \\
\left|c_{\alpha, n}\right\rangle & =\frac{\omega}{2 \pi} \int_{0}^{2 \pi / \omega} \mathrm{d} t\left|\phi_{\alpha}(t)\right\rangle \mathrm{e}^{\mathrm{i} n \omega t},
\end{aligned}
$$

it is obvious that the quasienergies come in classes, $\epsilon_{\alpha, n}=\epsilon_{\alpha}+n \omega, n=0, \pm 1, \pm 2, \ldots$, where each member corresponds to a physically equivalent solution. Therefore, all spectral information is contained in a single "Brillouin zone", $-\omega / 2 \leq \epsilon<\omega / 2$. 
By inserting the eigenstates (4) into the Schrödinger equation, Fourier expanding, and using the representation in the eigenstates of the unperturbed Hamiltonian, $H_{0}\left|\Psi_{k}\right\rangle=$ $E_{k}\left|\Psi_{k}\right\rangle$, a matrix eigenvalue equation [23,26],

$$
\sum_{n^{\prime}} \sum_{k^{\prime}} H_{n, k ; n^{\prime}, k^{\prime}} c_{n^{\prime}, k^{\prime}}=\epsilon c_{n, k}
$$

is derived, where

$$
\begin{aligned}
H_{n, k ; n^{\prime}, k^{\prime}}= & \left(E_{k}-n \omega\right) \delta_{n-n^{\prime}} \delta_{k-k^{\prime}} \\
& +S x_{k, k^{\prime}} \frac{\delta_{n-1-n^{\prime}}+\delta_{n+1-n^{\prime}}}{2}, \\
c_{n, k}= & \left\langle\Psi_{k} \mid c_{n}\right\rangle, \\
x_{k, k^{\prime}}= & \left\langle\Psi_{k^{\prime}}|x| \Psi_{k}\right\rangle .
\end{aligned}
$$

It is this matrix eigenvalue equation which we solve numerically to obtain the Floquet states and the quasienergies.

The invariance of the system under the generalized parity discussed in the previous section (see Eq. (2)) is of considerable help in the treatment of Eq. (6). The eigenvalue equations for the subspaces spanned by the even and odd eigenvectors decouple completely, and the matrices to be diagonalized take the block structure

$$
H_{\mathrm{e}}=\left(\begin{array}{ccccccc} 
& \vdots & \vdots & \vdots & \vdots & \vdots & \\
\cdots & E_{\mathrm{e}}+2 \omega I & X_{\mathrm{eo}} & 0 & 0 & 0 & \cdots \\
\cdots & X_{\mathrm{eo}} & E_{\mathrm{o}}+\omega I & X_{\mathrm{oe}} & 0 & 0 & \cdots \\
\cdots & 0 & X_{\mathrm{oe}} & E_{\mathrm{e}} & X_{\mathrm{eo}} & 0 & \cdots \\
\cdots & 0 & 0 & X_{\mathrm{eo}} & E_{\mathrm{o}}-\omega I & X_{\mathrm{oe}} & \cdots \\
\cdots & 0 & 0 & 0 & X_{\mathrm{oe}} & E_{\mathrm{e}}-2 \omega I & \cdots \\
\vdots & \vdots & \vdots & \vdots & \vdots &
\end{array}\right)
$$




$$
H_{\mathrm{o}}=\left(\begin{array}{ccccccc} 
& \vdots & \vdots & \vdots & \vdots & \vdots & \\
\cdots & E_{\mathrm{o}}+2 \omega I & X_{\mathrm{oe}} & 0 & 0 & 0 & \cdots \\
\cdots & X_{\mathrm{oe}} & E_{\mathrm{e}}+\omega I & X_{\mathrm{eo}} & 0 & 0 & \cdots \\
\cdots & 0 & X_{\mathrm{eo}} & E_{\mathrm{o}} & X_{\mathrm{oe}} & 0 & \cdots \\
\cdots & 0 & 0 & X_{\mathrm{oe}} & E_{\mathrm{e}}-\omega I & X_{\mathrm{eo}} & \cdots \\
\cdots & 0 & 0 & 0 & X_{\mathrm{eo}} & E_{\mathrm{o}}-2 \omega I & \cdots \\
& \vdots & \vdots & \vdots & \vdots & \vdots &
\end{array}\right)
$$

for the even (e) and the odd (o) subspace, respectively, where

$$
\begin{aligned}
& E_{\mathrm{e}}=\left(\begin{array}{cccc}
E_{0} & 0 & 0 & \cdots \\
0 & E_{2} & 0 & \cdots \\
0 & 0 & E_{4} & \cdots \\
\vdots & \vdots & \vdots & \ddots
\end{array}\right), \quad E_{\mathrm{o}}=\left(\begin{array}{cccc}
E_{1} & 0 & 0 & \cdots \\
0 & E_{3} & 0 & \cdots \\
0 & 0 & E_{5} & \cdots \\
\vdots & \vdots & \vdots & \ddots
\end{array}\right), \\
& X_{\mathrm{eo}}=\frac{1}{2}\left(\begin{array}{cccc}
x_{0,1} & x_{0,3} & x_{0,5} & \cdots \\
x_{2,1} & x_{2,3} & x_{2,5} & \cdots \\
x_{4,1} & x_{4,3} & x_{4,5} & \cdots \\
\vdots & \vdots & \vdots & \ddots
\end{array}\right), \quad X_{\mathrm{oe}}=\frac{1}{2}\left(\begin{array}{cccc}
x_{1,0} & x_{1,2} & x_{1,4} & \cdots \\
x_{3,0} & x_{3,2} & x_{3,4} & \cdots \\
x_{5,0} & x_{5,2} & x_{5,4} & \cdots \\
\vdots & \vdots & \vdots & \ddots
\end{array}\right) \text {, }
\end{aligned}
$$

All the matrices involved are formally infinite and have to be truncated according to the required numerical accuracy. Evidently, exploiting the generalized parity reduces the linear dimensions of vectors and matrices by a factor of two.

The tunnel doublets we are looking for are then composed of one state each from the even and the odd subspace, and the corresponding tunnel splittings are given by

$$
\Delta_{l}=\left|\epsilon_{l, \mathrm{o}}-\epsilon_{l, \mathrm{e}}\right|
$$

Due to its periodic, Brillouin-zone structure, the quasienergy spectrum gives no hint how to order the doublets. Instead, it is possible to associate a mean energy to the eigenstates by the relation 26] 


$$
\bar{E}_{\alpha}=\frac{\omega}{2 \pi} \int_{0}^{2 \pi / \omega} \mathrm{d} t\left\langle\psi_{\alpha}(t)|H(t)| \psi_{\alpha}(t)\right\rangle
$$

which is defined on the positive real axis and thus provides a basis for an ordering of the Floquet states. Using this order, we are able to pick out the doublets from the "groundstate" one upward to that immediately below the top of the barrier. In the following, we shall concentrate on this set of states, which for $D=8$ are 18 in number.

A quantity which facilitates making contact with the classical dynamics is the Husimi distribution [31,32]. It is defined as the overlap of a given state with a minimum-uncertainty (coherent) state localized at a position $(x, p)$ in phase space,

$$
Q_{\alpha}(x, p ; t)=\frac{1}{2 \pi \hbar}\left|\left\langle\xi \mid \psi_{\alpha}(t)\right\rangle\right|^{2}
$$

with

$$
|\xi\rangle=\mathrm{e}^{-|\xi|^{2} / 2} \sum_{n=0}^{\infty} \frac{\xi^{n}}{n !}|n\rangle,
$$

where $\xi=x+\mathrm{i} p$, and $|n\rangle$ is an eigenstate of the harmonic oscillator $\left(p^{2}+x^{2}\right) / 2$. The Husimi distribution represents a quantum state as a proper phase-space probability distribution with the highest resolution allowed by the uncertainty principle.

In particular, the Husimi distribution can be used to quantify the notion of an eigenstate residing predominantly in a specific (regular or chaotic) region of classical phase space. We define the mean overlap of a state $\left|\psi_{\alpha}(t)\right\rangle$ with the chaotic layer as

$$
\bar{\Gamma}_{\alpha}=\frac{\omega}{2 \pi} \int_{0}^{2 \pi / \omega} \mathrm{d} t \int_{-\infty}^{\infty} \mathrm{d} x \int_{-\infty}^{\infty} \mathrm{d} p Q_{\alpha}(x, p ; t) \Gamma(x, p ; t)
$$

Here, $\Gamma(x, p ; t)$ denotes the characteristic function for the chaotic region in the vicinity of the separatrix. Since the Husimi distribution forms a normalized probability distribution over phase space, we have $0 \leq \bar{\Gamma}_{\alpha} \leq 1$. The characteristic function can be determined numerically, e.g., by letting a trajectory started anywhere in this chaotic region "tick" boxes in a coarse-grained phase space of the desired resolution.

We start the discussion of our numerical results with a phenomenology of the Floquet states involved in driven tunneling. There are three aspects to be mentioned, the dependence 
on the quantum number (we choose the doublets 1 to 7 ), on the amplitude $S$ of the driving, and on time $t \bmod (2 \pi / \omega)$. For small enough $S$, e.g., $S=10^{-5}$, the Floquet states are nearly identical with the unperturbed eigenstates (Fig. 2). They are localized on tori with increasing characteristic action, embedded in the regular regions in the wells. The first qualitative changes to occur with $S$ taking larger values are due to the growth of the first resonance (see the previous section). For $S=10^{-2}$ (Fig. 3), the doublet states with $l=2,4,5$ take shapes that indicate their localization on tori that belong to the first resonance rather than to nonresonant motion in the wells; the characteristics of torus quantization remain. At $S=0.2$, the chaotic layer occupies an appreciable part of phase space. For the higherlying doublets, the distorted toroidal shape of the Husimi distribution then gives way to a more rugged form which less closely resembles a smeared-out torus (Fig. 4). A glance at the time dependence within one period complements these observations (Fig. 5): For a state pertaining to the first resonance, e.g., the two maxima of the Husimi distribution rotate clockwise, with the frequency of the driving, around the respective potential minima, as do the corresponding classical vortex tubes.

The central result of our study is presented in Fig. 6, where we compare the $S$ dependence of the tunnel splittings $\Delta_{l}$ (part a) with that of the overlaps $\bar{\Gamma}_{l, \mathrm{e}}$ (part b; we could quite as well have chosen $\left.\bar{\Gamma}_{l, \mathrm{O}}\right)$. Looking at the tunnel splittings first, a relatively clear-cut picture emerges: For $S \lesssim 10^{-3}$, the tunnel splittings do not deviate significantly from their unperturbed values (apart from an irregularity in $\Delta_{2}$ which roughly coincides with a region of rapid growth of the first resonance). They increase roughly exponentially with the quantum number $l$, from a value $\Delta_{1}=5.20 \times 10^{-18}$, in good agreement with the corresponding semiclassical (instanton-method, cf. ref. [33]) estimate [12] $\Delta_{1}^{\mathrm{sc}}=\sqrt{128 D / \pi} \exp (-16 D / 3)=5.33 \times 10^{-18}$, to $\Delta_{8}=5.59 \times 10^{-4}$. For $S \gtrsim 10^{-3}$, the splittings start one by one to grow exponentially with $S$, from the ground-state doublet $(l=1)$ upwards, so that at $S=0.2$, the range of the splittings has shrunk from 14 to three orders of magnitude. The $S$ dependence of the overlaps shows the same qualitative features as that of the tunnel splittings: There is no significant deviation from the unperturbed values for $S \lesssim 10^{-3}$, while for larger $S$, exponential 
growth sets in from bottom to top of the doublet ladder, so that the $\bar{\Gamma}_{l, \mathrm{e}}$ successively join in an approximately single line.

The qualitative agreement between the two respective groups of functional dependences indicates that there exists a strong correlation between the tunnel splittings and the overlaps with the chaotic layer. Furthermore, the steep exponential increase occurring in both quantities coincides with the onset of chaotic motion in the classical dynamics, whereas, e.g., the transfer of phase-space area from nonresonant motion to the first resonance hardly leaves any trace in the tunnel splittings. Insofar, the simple picture sketched in the Introduction is confirmed. Details of our expectation, however, need to be revised.

In particular, the notion that each splitting widens up individually as the corresponding quantizing torus resolves, is not unambiguously corroborated by the data. It would imply that the transitions to a large splitting occur from top to bottom, i.e., first for the doublet localized on the outermost torus, the one with the highest mean energy. Indeed, if this transition is assessed from the splittings passing a certain absolute threshold, say $\Delta_{l}=10^{-4}$, that order is roughly followed (at least for those doublets we can keep track of that far). If, however, the point of onset of exponential growth, visible in a logarithmic plot, is taken as the criterion, the order is reversed.

Another remarkable fact is that the widening of the splittings, and the concomitant change in character of the eigenfunctions, as a function of $S$, is a continuous process that can only vaguely be associated with the decay of a KAM torus, taken as as a discrete event. Even doublet states overlapping by $70 \%$ with the chaotic layer may still show a relatively small splitting and exhibit the signature of a regular state in their spatial structure and time dependence (see, e.g., Figs. 4 and 7). It remains to be clarified whether this retarded decay of the tunnel doublets corresponds to the gradual disintegration of classical tori via cantori and vague tori.

Unfortunately, our numerical means do not allow to extend the data beyond $S=0.2$. Therefore, the eventual saturation of the growth of the tunnel splittings, when they reach the order of magnitude of the mean level spacing, could not be studied. Furthermore, even 
if the double well with a scaled barrier height $D=8$ is the closest approach to the classical limit we could practically afford, the quantal dynamics of this system is not yet sensitive enough for the details of the classical phase space to allow for a conclusive numerical test of a semiclassical description.

\section{SUMMARY}

In which way does the onset of classical chaos in the vicinity of the separatrix influence the tunnel splittings in a periodically driven bistable system? The random-matrix theory for mixed (chaotic and regular) systems, together with semiclassical considerations, suggest the following simple answer: A tunnel splitting undergoes a transition from exponentially small values to a size comparable with the mean level spacing, as the corresponding pair of symmetry-related, quantizing tori resolve in a common chaotic region. In order to test this hypothesis, we quantified the association of an eigenstate to the chaotic layer along the separatrix in terms of the overlap of its Husimi representation with this phase-space region. We found a striking qualitative agreement between the functional dependences of the splittings and the corresponding overlaps on the amplitude of the driving force. Both groups of quantities show transitions to steep exponential growth as the chaotic layer along the separatrix spreads at the expense of the regular regions around the potential wells. The above hypothesis does, however, imply more, namely that the transitions to a large splitting for the individual doublets occur in a specific order given by the fate of the corresponding tori. For the driven double well this order is roughly from top to bottom on the energy axis (where "energy" should be read as "mean energy"). This is indeed what we observe if the widening of the splittings is assessed from their growing beyond some absolute threshold value. If, however, the onset of exponential growth is taken as the criterion, the order turns out to be from bottom to top. As a further unexpected detail, the regular character of doublet states survives until far into a parameter region where they are already located amidst the chaotic layer. 
On first sight it may appear strange that a static quantity like the overlap with the chaotic layer should bear on a transport phenomenon such as tunneling. From a semiclassical point of view, this may become understandable if one assumes that a path-integral expression for the tunnel splitting becomes dominated by contributions from paths which pass through the chaotic layer, as soon as chaotic diffusion enables significant classical transport between the wells. Our study, which is intended solely as a first numerical survey, should encourage to cast this notion in a precise semiclassical form. Related topics which deserve further investigation are the time-domain aspects of tunneling through the chaotic layer, the occurrence of crossings between tunnel doublets and their influence on the dynamics. Another interesting open question is whether states which reside within the chaotic layer but appear regular in character, can be associated with vague tori of the classical dynamics. 


\section{REFERENCES}

[1] G. M. Zaslavsky, Sov. Phys.-Usp. 22, 788 (1979) [G. M. Zaslavsky, Usp. Fiz. Nauk 129, $211(1979)]$.

[2] O. Bohigas and M.-J. Giannoni, Lecture Notes in Physics vol.209 (Springer, Berlin, 1984), p. 1.

[3] M. V. Berry, Proc. R. Soc. A 400, 229 (1985).

[4] G. Casati, B. V. Chirikov, F. M. Izrailev, and J. Ford, Lecture Notes in Physics vol.93 (Springer, Berlin, 1979), p. 334.

[5] S. Fishman, R. E. Prange, and D. R. Grempel, Phys. Rev. Lett. 42, 509 (1982); Phys. Rev. A 29, 1639 (1984).

[6] D. L. Shepelyansky, Phys. Rev. Lett. 56, 677 (1986).

[7] M. J. Davis and E. J. Heller, J. Chem. Phys. 75, 246 (1986).

[8] O. Bohigas, S. Tomsovic, and D. Ullmo, Phys. Rev. Lett. 64, 1479 (1990); Phys. Rev. Lett. 65, 5 (1990).

[9] S. Tomsovic and D. Ullmo, Tunneling in the Presence of Chaos, preprint, 1990.

[10] O. Bohigas, S. Tomsovic, and D. Ullmo, Phys. Rep. 223, 43 (1993).

[11] W. A. Lin and L. E. Ballentine, Phys. Rev. Lett. 65, 2927 (1990); Phys. Rev. A 45, 3637 (1992).

[12] F. Grossmann, P. Jung, T. Dittrich, and P. Hänggi, Z. Phys. B 84, 315 (1991); Phys. Rev. Lett. 67, 516 (1991).

[13] F. Grossmann, T. Dittrich, P. Jung, and P. Hänggi, J. Stat. Phys. 70, 229 (1993).

[14] F. Grossmann, T. Dittrich, and P. Hänggi, Physica B 175, 293 (1991).

[15] J. Plata and J. M. Gomez Llorente, J. Phys. A 25, L303 (1992). 
[16] M. Wilkinson, Physica 21D, 341 (1986); J. Phys. A 20, 635 (1987).

[17] M. Wilkinson and J. H. Hannay, Physica 27D, 201 (1987).

[18] R. Utermann, T. Dittrich, and P. Hänggi, in Proceedings of the XXth International Conference on Low Temperature Physics, Eugene, 1993, edited by R. J. Donnelly (NorthHolland - Elsevier, Amsterdam, 1993).

[19] H. P. Breuer and M. Holthaus, Ann. Phys. (New York) 211, 249 (1991).

[20] M. V. Berry and M. Robnik, J. Phys. A 17, 2413 (1984).

[21] L. E. Reichl, in The Transition to Chaos: In Conservative and Classical Systems: Quantum Manifestations (Springer, New York, 1992), Chaps. 3.9 and 9.5.1, and refs. therein.

[22] W. P. Reinhardt, J. Phys. Chem. 86, 2158 (1982); R. B. Shirts and W. P. Reinhardt, J. Chem. Phys. 77, 5204 (1982).

[23] J. H. Shirley, Phys. Rev. 138B, 979 (1965).

[24] H. Sambe, Phys. Rev. A 7, 2203 (1973).

[25] N. L. Manakov, V. D. Ovsiannikov, and L. P. Rapoport, Phys. Rep. 141, 319 (1986).

[26] S. Chu, Adv. Chem. Phys. 73, 739 (1989).

[27] A. Peres, Phys. Rev. Lett. 67, 158 (1991).

[28] L. E. Reichl and W. M. Zheng, in Directions in Chaos, vol.1, edited by H. B. Lin (World Scientific, Singapore, 1987), p. 17.

[29] A. J. Lichtenberg and M. A. Lieberman, Regular and Stochastic Motion, Vol. 38 of Appl. Math. Sci. (Springer, New York, 1983).

[30] H. P. Breuer, K. Dietz, and M. Holthaus, Physica D 46, 317 (1990).

[31] K. Husimi, Proc. Phys. Math. Soc. Jap. 22, 264 (1940). 
[32] R. J. Glauber, in Quantum Optics, edited by A. Maitland (Academic, London, 1970).

[33] S. Coleman, in The Whys of Subnuclear Physics, Proceedings of the International School of Subnuclear Physics, Erice, 1977, edited by A. Zichichi (Plenum, New York, 1979). 


\section{FIGURES}

FIG. 1. Classical phase-space portraits of the periodically driven double well at phase 0 of the driving, for various values of the driving amplitude. The parameter values are $D=8, \omega=0.95$, and (a) $S=0$, (b) $S=10^{-3}$, (c) $S=0.02$, (d) $S=0.2$.

FIG. 2. Contour plots of the Husimi distributions for the Floquet states $\left|\psi_{2, \mathrm{e}}(0)\right\rangle$ (a) and $\left|\psi_{7, \mathrm{e}}(0)\right\rangle(\mathrm{b})$, at $S=10^{-5}$.

FIG. 3. Contour plot of the Husimi distribution for the Floquet state $\left|\psi_{5, \mathrm{e}}(0)\right\rangle$ at $S=0.02$. It should be compared with the corresponding classical phase-space portrait, Fig. 1c.

FIG. 4. Contour plot of the Husimi distribution for the Floquet state $\left|\psi_{7, \mathrm{e}}(0)\right\rangle$ at $S=0.2$.

FIG. 5. Contour plot of the Husimi distribution for the Floquet state $\left|\psi_{5, \mathrm{e}}(t)\right\rangle$ at $S=0.02$ and (a) $\omega t=\pi / 4$ and (b) $\omega t=\pi / 2$ (b), compared to the corresponding classical phase-space portraits at the same parameter values and phases (parts (c) and (d), respectively). For the initial states $(\omega t=0)$, see Figs. 1c and 3, respectively. The states at later times $\omega t=n \pi / 4$ are related to those shown by simple phase-space symmetries, as implied by the generalized parity, Eq. (2).

FIG. 6. Tunnel splittings (a) and overlaps with the chaotic layer (b) for the seven lowest tunnel doublets, as functions of the amplitude of the driving.

FIG. 7. Contour plot of the Husimi distribution for the Floquet state $\left|\psi_{7, \mathrm{e}}(t)\right\rangle$ at $S=0.02$ and (a) $\omega t=\pi / 4$ and (b) $\omega t=\pi / 2$. For the initial state, see Fig. 4 . The states at later times $\omega t=n \pi / 4$ are related by simple phase-space symmetries to those shown here, as implied by the generalized parity,Eq. (2). 